\title{
UNCONDITIONALLY POSITIVE FINITE DIFFERENCE AND STANDARD EXPLICIT FINITE DIFFERENCE SCHEMES FOR POWER FLOW EQUATION
}

\author{
BRANKO DRLJAČA ${ }^{1 *}$, SVETISLAV SAVOVIĆ \\ ${ }^{1}$ Faculty of Sciences, University of Priština, Kosovska Mitrovica, Serbia \\ ${ }^{2}$ Faculty of Sciences, University of Kragujevac, Kragujevac, Serbia
}

\begin{abstract}
Power flow equation for step-index glass optical fiber was solved using recently reported unconditionally-positive finite difference (UPFD) scheme. Solution obtained using UPFD scheme was compared with solution obtained using standard explicit finite difference (EFD) scheme. For accuracy testing both schemes were compared with analytical solution for steady state distribution of given fiber. The advantage of UPFD is reflected in stability of the scheme regardless of discretization step taken. Nevertheless EFD scheme has better concurrence with analytical solution than UPFD. This is due to the additional truncation-error terms in the approximations of the first and second derivatives with respect to $\theta$.
\end{abstract}

Keywords: Parabolic equation, Numerical schemes, Step index optical fiber.

\section{INTRODUCTION}

Partial differential equations are used to model a wide variety of problems in natural sciences. Usually those are physical, chemical, biological and engineering problems such as transmission of light through optical fibers, heat transfer, transport and reaction of chemical species, solid state physics, adsorption of pollutants in soil and diffusion of radon and neutrons (Bear et al., 2007; Hetrick, 1971; Kevkic et al., 2019; Murray, 2002; Petrović, 2017; Shih, 1984). Often those equations are parabolic differential equations with no, or limited, analytical solution and their solvation requires various numerical techniques (Djordjevich 2013; Savović et al., 2009; Savović et al., 2012; Savović et al., 2013a; Savović et al., 2013b). In case of three-dimensional problems solutions are often obtained by the finite element method (Urošević et al., 2003). If problems are one-dimensional they are much easier solved by finite difference method (Savović et al., 2013b). Both methods have advantages and limitations, and the choice between two methods is mostly conditioned by geometry of the problem. For neither does the standard finite discretization explicitly constrain the solutions to positive values, which may lead to numerical instabilities and oscillations of the solution. Moreover there is no unanimous opinion of choice between different finite difference methods for diverse applications.

With the development of computers most algorithms for numerical methods were implemented in a variety of programming languages. In the beginning, during 1970s and 1980s, implicit finite difference methods (IFDMs) were generally first choice. Being often unconditionally stable, the IFDM allows larger step lengths. Despite, this does not increase
IFDM's computational efficiency because extremely large matrices must be manipulated at each calculation step. With further advancement of the computers this trend has been changing, shifting the emphasis to explicit finite difference methods (EFDMs). Although EFDM is not unconditionally stable we found that it is also simpler in addition to being computationally more efficient (Savović et al., 2009; Savović et al., 2012; Simovic et al., 2014).

The constant development of numerical methods in search of the best possible solution has led to the proposition of new solutions. Several authors propose new numerical solution of the parabolic differential equations that guarantees positivity of the solutions and that is independent both of the step size in $z$ direction and mesh size (Chen-Charpentier et al., 2013; Liu et al., 2010; Quang et al., 2006). The method works with reaction terms that are the sum of a positive function and a negative function of the unknown - either or both may be zero. It is applicable to both, problems where either advection or diffusion dominates. In this work, a recently reported UPFD scheme and a standard EFD scheme for solving power flow equation are compared to analytical solution for steady-state distribution for glass optical fiber CGW-CGE-68 (Drljaca, 2011).

\section{THEORETICAL PART}

Power distribution in optical fiber can be determined by using one of the three methods: ray-tracing method, wave approach and Gloge's power flow equation. The simplest way that can describe evolution of the power in the multimode optical fibers and account for all important characteristics of the fiber is Gloge`s power flow equation. The Gloge's power flow equation is given as (Gloge, 1972):

\footnotetext{
* Corresponding author: branko.drljaca@ pr.ac.rs 


$$
\frac{\partial P(\theta, z)}{\partial z}=-\alpha(\theta) P(\theta, z)+\frac{D}{\theta} \frac{\partial}{\partial \theta}\left(\theta \frac{\partial P(\theta, z)}{\partial \theta}\right)
$$

where $P(\theta, z)$ is the angular power distribution, $z$ is distance from the input end of the fiber, $\theta$ is the propagation angle with respect to the core axis, $D$ is the coupling coefficient assumed constant (Garito et al. 1998; Savovic et al., 2015) and $\alpha(\theta)$ is the modal attenuation. The boundary conditions are $P\left(\theta_{c}, z\right)=0$, where $\theta_{c}$ is the critical angle of the fiber, and $D(\partial P / \partial \theta)=0$ at $\theta=0$. Condition $P\left(\theta_{c}, z\right)=0$ implies that modes with infinitely high loss do not carry power. Condition $D(\partial P / \partial \theta)=0$ at $\theta=0$ indicates that the coupling is limited to the modes propagating with $\theta>0$. After simplification equation (1) becomes (Djordjevich et al., 2000):

$$
\frac{\partial P(\theta, z)}{\partial z}=\frac{D}{\theta} \frac{\partial P(\theta, z)}{\partial \theta}+D \frac{\partial^{2} P(\theta, z)}{\partial \theta^{2}}
$$

Numerical solution of equation (2) is obtained by EFDM, used in our previous works (Drljaca, 2011; Savović et al. 2013a; Savovic et al., 2015) and UPFDM proposed by other authors (Chen-Charpentier et al., 2013; Liu et al., 2010; Quang et al., 2006.]. The results thus obtained are compared to the analytical solution of steady-state distribution for the given glass optical fiber:

$$
P(\theta, z)=J_{0}\left(2.405 \frac{\theta}{\theta_{c}}\right) \exp \left(-\gamma_{0} z\right)
$$

where $J_{0}$ is the Bessel function of the first kind and zero order and $\gamma_{0}\left[\mathrm{~m}^{-1}\right]=2.405^{2} \mathrm{D} / \theta_{c}^{2}$ is the attenuation coefficient (Drljaca, 2011).

\section{NUMERICAL METHOD}

In order to compare results obtained by EFD and UPFD method we considered equation (2) for the same input data used previously (Drljaca, 2011), subjected to following initial and boundary conditions: $P\left(\theta_{c}, \mathrm{z}\right)=0 ; D \frac{\partial P\left(\theta_{=0}, z\right)}{\partial \theta}=0 \quad$ (Djordjevich et. al. 2000; Drljaca, 2011; Savović et al., 2013b; Savović et al., 2015; Simovic et al., 2014).

\section{Standard Explicit Finite Difference Method}

After applying EFD scheme (Anderson, 1995):

$$
\begin{aligned}
& \left(\frac{\partial P(\theta, \mathrm{z})}{\partial \theta}\right)_{m, n}=\frac{P_{m+1, n}-P_{m-1, n}}{2 \Delta \theta} \\
& \left(\frac{\partial^{2} P(\theta, \mathrm{z})}{\partial \theta^{2}}\right)_{m, n}=\frac{P_{m+1, n}-2 P_{m, n}+P_{m-1, n}}{(\Delta \theta)^{2}} \\
& \left(\frac{\partial P(\theta, \mathrm{z})}{\partial z}\right)_{m, n}=\frac{P_{m, n+1}-P_{m, n}}{\Delta z}
\end{aligned}
$$

equation (2) becomes:

$$
\begin{aligned}
\frac{P_{m, n+1}-P_{m, n}}{\Delta z}= & \frac{D}{\theta} \frac{P_{m+1, n}-P_{m-1, n}}{2 \Delta \theta}+ \\
& +D \frac{P_{m+1, n}-2 P_{m, n}+P_{m-1, n}}{(\Delta \theta)^{2}}
\end{aligned}
$$

Truncation errors of the scheme are $O\left(\Delta \theta^{2}, \Delta z\right)$.

When (5) is rewritten in explicit form it becomes:

$$
\begin{aligned}
P_{m, n+1}= & P_{m, n}+\frac{\Delta z D}{\theta} \frac{P_{\mathrm{m}, n+1}-P_{m-1, n}}{\Delta \theta}+ \\
& +\Delta z D \frac{P_{m+1, n}-2 P_{\mathrm{m}, n+1}+P_{m-1, n}}{\Delta \theta^{2}}
\end{aligned}
$$

Unconditionally Positive Finite Difference Method

After applying UPFD scheme:

$$
\begin{aligned}
& \left(\frac{\partial P(\theta, \mathrm{z})}{\partial \theta}\right)_{m, n}=\frac{P_{m, n+1}-P_{m-1, n}}{\Delta \theta} \\
& \left(\frac{\partial^{2} P(\theta, \mathrm{z})}{\partial \theta^{2}}\right)_{m, n}=\frac{P_{m+1, n}-2 P_{m, n+1}+P_{m-1, n}}{(\Delta \theta)^{2}} \\
& \left(\frac{\partial P(\theta, \mathrm{z})}{\partial z}\right)_{m, n}=\frac{P_{m, n+1}-P_{m, n}}{\Delta z}
\end{aligned}
$$

equation (2) becomes:

$$
\frac{P_{m, n+1}-P_{m, n}}{\Delta z}=\frac{D}{\theta} \frac{P_{\mathrm{m}, n+1}-P_{m-1, n}}{\Delta \theta}+D \frac{P_{m+1, n}-2 P_{\mathrm{m}, n+1}+P_{m-1, n}}{\Delta \theta^{2}}
$$

When equation (8) is rewritten in explicit form it becomes:

$$
P_{m, n+1}=\frac{\hat{D} P_{m+1, n}+P_{m, n} / \Delta z+(\hat{u}+\hat{D}) \mathrm{P}_{m-1, n}}{1 / \Delta z+\hat{u}+2 \hat{D}}
$$

where $\hat{u}=-D / \Delta \theta \cdot \theta$ and $\hat{D}=D / \Delta \theta^{2}$. If the parameters $D / \theta$, and $D$ are all non-negative, and therefore $\hat{u}$ and $\hat{D}$ are both positive, then the numerical scheme represents a UPFD method for any $\Delta z>0$ and $\Delta \theta>0$. In this way, the solutions of the scheme are always positive independently of the choice of space and angle steps [Chen-Charpentier et al., 2013.]. If $D / \theta$ $<0$, Chen-Charpentier and Kojouharov (Chen-Charpentier et al., 2013 ) proposed that this term is discretized as $+\frac{D}{\theta} \frac{P_{m, n}-P_{m-1, n+1}}{\Delta x}$ so that the UPFD solution would remain positive. In that case power flow equation is written in the following form: 


$$
\begin{aligned}
P_{m, n+1}= & \frac{\hat{D} \Delta \theta^{2} P_{m+1, n}+P_{m, n}(1 / \Delta z-\hat{u})}{1 / \Delta z+2 \hat{D}}+ \\
& +\frac{\hat{D} \mathrm{P}_{m-1, n}+\hat{u} \mathrm{P}_{m-1, n+1}}{1 / \Delta z+2 \hat{D}}
\end{aligned}
$$

Truncation error of the scheme is $O(\Delta \theta+\Delta z)$

The method (7) proposed by Chen-Charpentier and Kojouharov (Chen-Charpentier et al., 2013) is simultaneously explicit and unconditionally positive, but it is not unconditionally consistent. There are extra truncation error terms because the approximations of the first and second derivatives with respect to $\theta$ are evaluated at different lengths. One way to reduce this error is to choose the length step depending on the mesh size so that the inconsistent terms approach zero when the mesh is refined. Second approach is to incorporate these terms into the numerical scheme to achieve a consistent approximation of the original partial differential equation (Chen-Charpentier et al., 2013).

\section{RESULTS}

In order to present obtained far-field intensity patterns for numerical calculations we used same fiber that was previously used in different experiments and numerical simulations - CGWCGE-68 glass optical fiber. The fiber has critical angle $\theta_{c}=7.26^{\circ}$ measured inside the fiber and $\theta_{c}=10.6^{\circ}$ measured in air. Coupling coefficient for this fiber is $D=7.9 \times 10^{-7} \mathrm{rad}^{2} / \mathrm{m}$ at room temperature and was used for numerical calculations (Drljaca, 2011). Attenuation coefficient is calculated as $\gamma_{0}=2.86 \times 10^{-4} \mathrm{~m}^{-1}$.

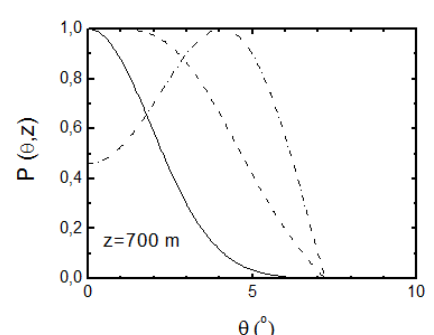

a)

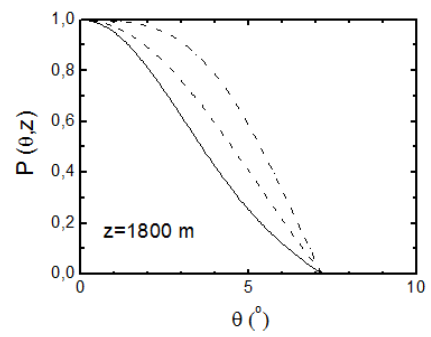

c)

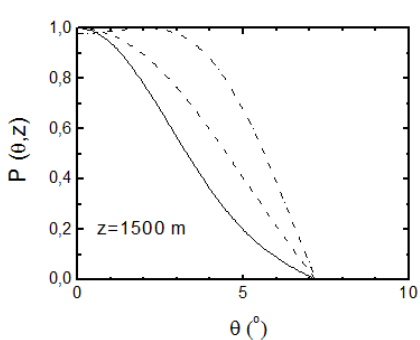

b)

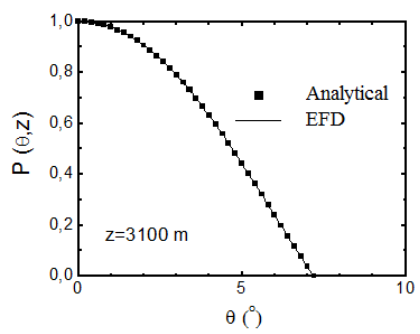

d)
Figure 1. Normalized output angular power distribution obtained by EFD method at different locations along the CGW-CGE-68 fiber for three Gaussian input angles $\theta_{0}=0 \circ$ (solid line), $3^{\circ}$ (dashed line) and $6^{\circ}$ (dotted line) at: (a) $z=700 \mathrm{~m}$; (b) $z=1500$ $\mathrm{m} ;$ (c) $z=1800 \mathrm{~m}$ and (d) $z=3100 \mathrm{~m}$. [Drljaca, 2011.].
Figure 1. shows normalized far field patterns for different lengths and three different launch angles $\left(\theta_{0}=0,3\right.$ and $\left.6^{\circ}\right)$ obtained by standard EFD method, and analytical solution of power flow equation (2) (Drljaca, 2011). In Figure 2. numerical solutions obtained by UPFD method are presented.

As could be seen from Fig. 1 and Fig. 2 that both EFD and UPFD methods have a good agreement with analytical solution of equation (2). However it could be seen that UPFD scheme is less accurate than EFD scheme when compared to analytical solution. This is due to additional truncation error that occurs in UPFD scheme.

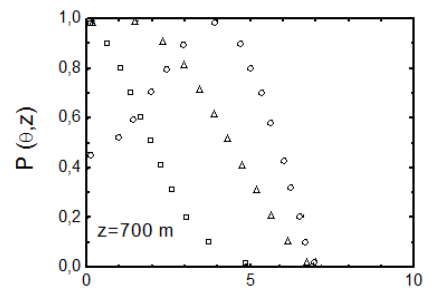

$\theta\left(^{\circ}\right)$

a)

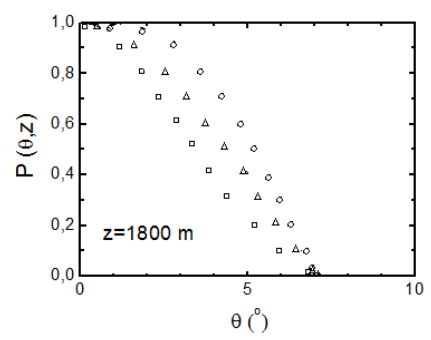

c)

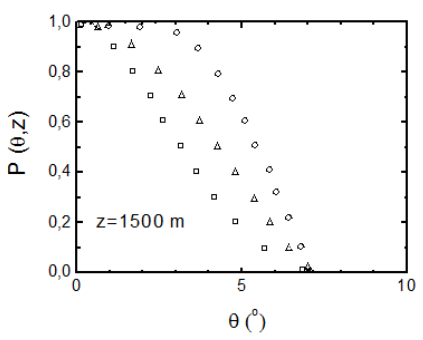

b)

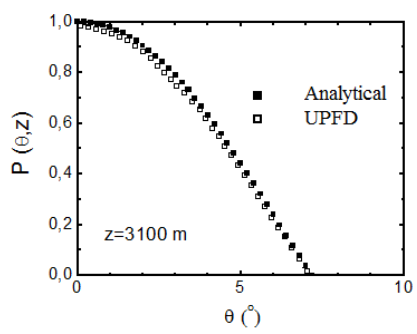

Figure 2. Normalized output angular power distribution obtained by UPFD method at different locations along the CGW-CGE-68 fiber for three Gaussian input angles $\theta_{0}=0^{\circ}$ (squares), $3^{\circ}$ (triangles) and $6^{\circ}$ (circles) at: (a) $z=700 \mathrm{~m}$; (b) $z=1500 \mathrm{~m}$; (c) $z=1800 \mathrm{~m}$ and $(\mathrm{d}) z=3100 \mathrm{~m}$.

\section{CONCLUSION}

Standard explicit finite difference (EFD) scheme and recently reported unconditionally positive finite difference (UPFD) scheme for solving parabolic differential equations are compared to previously obtained analytical solution of power flow equation. We have shown that this scheme is less accurate, even though UPFD scheme guarantees the positivity of the solutions for arbitrary step sizes, and is stable. The reason for this is additional truncation error in the approximations to the first and second derivatives with respect to $\theta$, which are evaluated at different lengths, which is contained in the UPFD scheme. If the accuracy of the numerical scheme over stability is needed we propose usage of EFD scheme before UPFD scheme. 


\section{ACKNOWLEDGMENTS}

The work described in this paper was supported by the grant from the Serbian Ministry of Education and Science (Project no. 171011).

\section{REFERENCES}

Anderson, J. D. 1995. Computational fluid dynamics. New York: McGraw-Hill.

Bear, J. 2007. Hydraulics of Groundwater. Dover - Minneola.

Chen-Charpentier, B. M., \& Kojouharov, H. V. 2013. An unconditionally positivity preserving scheme for advectiondiffusion reaction equations. Mathematical and Computer Modelling, 57(9-10), pp. 2177-2185. doi:10.1016/j.mcm.2011.05.005

Dang, Q. A., \& Ehrhardt, M. 2006. Adequate numerical solution of air pollution problems by positive difference schemes on unbounded domains. Mathematical and Computer Modelling, 44(9-10), pp. 834-856. doi:10.1016/j.mcm.2006.02.016

Djordjevich, A., \& Savović, S. 2000. Investigation of mode coupling in step index plastic optical fibers using the power flow equation. IEEE Photonics Technology Letters, 12(11), pp. 1489-1491. doi:10.1109/68.887704

Djordjevich, A., \& Savović, S. 2013. Solute transport with longitudinal and transverse diffusion in temporally and spatially dependent flow from a pulse type source. International Journal of Heat and Mass Transfer, 65, pp. 321326. doi:10.1016/j.ijheatmasstransfer.2013.06.002

Drljaca, B. 2011. Modelovanje prostiranja svetlosti kroz višemodna optička vlakna sa stepenastim indeksom prelamanja primenom jednačine protoka snage. Kragujevac: PMF Kragujvac.

Garito, A. F., Wang, J., \& Gao, R. 1998. Effects of Random Perturbations in Plastic Optical Fibers. Science, 281(5379), pp. 962-967. doi:10.1126/science.281.5379.962

Gloge, D. 2013. Optical Power Flow in Multimode Fibers. Bell System Technical Journal, 51(8), pp. 1767-1783. doi:10.1002/j.1538-7305.1972.tb02682.x

Hetrick, D. K. 1971. Dynamics of Nuclear Reactors. Chicago: University of Chicago.

Kevkic, T., Stojanovic, V., \& Petkovic, D. 2019. Solving Schrödinger Equation for a Particle in One-Dimensional Lattice: An Homotopy Perturbations Approach. Romanian Reports in Physics, 71(101).
Liu, L., Clemence, D. P., \& Mickens, R. E. 2010. A nonstandard finite difference scheme for contaminant transport with kinetic Langmuir sorption. Numerical Methods for Partial Differential Equations, 27(4), pp. 767-785. doi:10.1002/num.20551

Murray, J. D. 2002. Mathematical Biology I. Berlin: SpringerVerlag.

Petrović, M., Kontrec, N., \& Panić, S. 2017. Determination of accelerated factors in gradient descent iterations based on Taylor's series. The University Thought - Publication in Natural Sciences, 7(1), pp. 41-45. doi:10.5937/univtho714337

Savović, S., \& Caldwell, J. 2009. Numerical solution of Stefan problem with time-dependent boundary conditions by variable space grid method. Thermal Science, 13(4), pp. 165174. doi:10.2298/tsci0904165s

Savović, S., Djordjevich, A., \& Ristić, G. 2012. Numerical solution of the transport equation describing the radon transport from subsurface soil to buildings. Radiation Protection Dosimetry, 150(2), pp. 213-216. doi: $10.1093 / \mathrm{rpd} / \mathrm{ncr} 397$

Savović, S., Drljača, B., \& Djordjevich, A. 2013. Influence of launch-beam distribution on bandwidth in step-index plastic optical fibers. Applied Optics, 52(6), p. 1117 doi:10.1364/ao.52.001117

Savović, S., \& Djordjevich, A. 2013. Numerical solution for temporally and spatially dependent solute dispersion of pulse type input concentration in semi-infinite media. International Journal of Heat and Mass Transfer, 60, pp. 291-295. doi:10.1016/j.ijheatmasstransfer.2013.01.027

Savovic, S., Kovacevic, M. S., Bajic, J. S., Stupar, D. Z., Djordjevich, A., Zivanov, M., Drljaca, B., Simovic, A., \& Oh, K. 2015. Temperature Dependence of Mode Coupling in low-NA Plastic Optical Fibers. Journal of Lightwave Technology, 33(1), pp. 89-94. doi:10.1109/jlt.2014.2375515

Shih, T. 1984. Numerical Heat Transfer. Berlin: Springer-Verlag.

Simović, A., Savović, S., Drljača, B., \& Djordjevich, A. 2014. Influence of intermediate layer on transmission characteristics of W-type optical fibers. Optics and Laser Technology, 57, pp. 209-215. doi:10.1016/j.optlastec.2013.10.024

Urošević, V., \& Nikezić, D. 2003. Radon transport through concrete and determination of its diffusion coefficient. Radiat. Prot. Dosim, 104, pp. 65-70. 